\title{
Electrification of Dielectric Satellites under the Influence of Electron Flows of the Earth's Radiation Belts
}

\author{
V. V. Surkov ${ }^{a, b}$ * and K. S. Mozgov ${ }^{c}$ \\ ${ }^{a}$ Pushkov Institute of Terrestrial Magnetism, Ionosphere, and Radio Wave Propagation, Moscow, Troitsk, 108840 Russia \\ ${ }^{b}$ Schmidt Institute of Physics of the Earth, Russian Academy of Sciences, Moscow, 123995 Russia \\ c JSC “Research-and-Production Corporation "Precision Systems and Instruments", Moscow, Russia \\ *e-mail: surkovvadim@yandex.ru \\ Received November 3, 2020; revised December 7, 2020; accepted January 28, 2021
}

\begin{abstract}
The effect of electron fluxes from the Earth's radiation belts on satellites made of dielectric materials is studied theoretically. Spherical shaped nanosatellites of the BLITS and BLITS-M types are considered as a model. An analytical solution is obtained for the dependence of the electric field on the distance to the center of the satellite. Empirical formulas are used for the electron flux density and the track length in matter depending on the electron energy. The energy losses of incident electrons in the Debye shielding layer that surrounds the satellite, as well as the appearance of radiation conductivity in the surface layer of the dielectric, are taken into account. The reasons for the nonmonotonic dependence of the electric field on the satellite radius are established. Despite the fact that the electric field inside the satellite is smaller than the electrical breakdown threshold of the solid dielectric, it can be assumed that the dielectric micro-breakdown can occur in the surface layer of the dielectric and near inhomogeneities.
\end{abstract}

DOI: $10.1134 / \mathrm{S} 0016793221040174$

\section{INTRODUCTION}

An important factor affecting the environment on spacecraft (SC) is the fluxes of high-energy electrons and ions, which can deeply penetrate the thickness of materials and the inner parts of the spacecraft (Kuznetsov, 2007; Lai, 2011). As a result of this effect, the SC acquires electric charges that are distributed over the surfaces of conducting structures and the thickness of dielectric materials. The process of the acquisition of electric charges by materials significantly depends on their conductivity and secondary emission processes, which, in turn, are subject to changes under the influence of cosmic rays and other factors of outer space. However, the nature of these changes is still poorly understood. Analysis of the electrification of a real SC is a difficult problem, since the design of modern SC contains a large number of conducting and dielectric materials. Potential differences arise among them, reaching tens of kilovolts in some cases (Novikov et al., 2007; Lai and Cahoy, 2017; Bezrodnykh et al., 2016; Lai et al., 2018). When dielectrics are irradiated by relativistic electrons with energies 1-10 MeV characteristic of the Earth's radiation belts (ERBs), the depth of their penetration into the dielectric exceeds several millimeters; this creates a risk of electrical breakdown and material destruction (Akishin and Novikov, 1985). The electric discharges in dielectric samples, which were observed in scien- tific experiments onboard the CRESS SC, are presumably caused by the electron fluxes of the ERB (Weber, 1964). As a result of the formation of discharge channels, the optical and mechanical properties of dielectric materials can sharply deteriorate. In addition, electric discharges occurring on the surface and inside the SC body are an important reason for the appearance of malfunctions and failures in the operation of the equipment onboard the SC (DeForest, 1972; Novikov et al., 2007; Lai, 2011; Bezrodnykh et al., 2016; Lai and Cahoy, 2017; Lai et al., 2018).

Numerical simulation are usually used for the mathematical modeling of the electrification of modern SC structures (Lai, 2011; Novikov et al., 2017). Analytical results can be obtained for some SC with a relatively simple spherical configuration. These include the passive laser satellites Larets, BLITS, GFZ-1, and WESTPAC, which are designed for the calibration of ground-based measuring instruments with the use of high-precision laser measurements (Surkov and Mozgov, 2019). In particular, the Ball Lens In The Space (BLITS) nanosatellite, which was launched in September 2009 into a circular sun-synchronous orbit with an inclination of $98.77^{\circ}$ and a height of $832 \mathrm{~km}$, was made in the form of a glass sphere according to the principle of an optical Luneberg lens (Kucharski et al., 2011; Vasiliev, 2018). Structurally, it consisted of two glass hemispheres glued to a glass ball lens. A reflective 


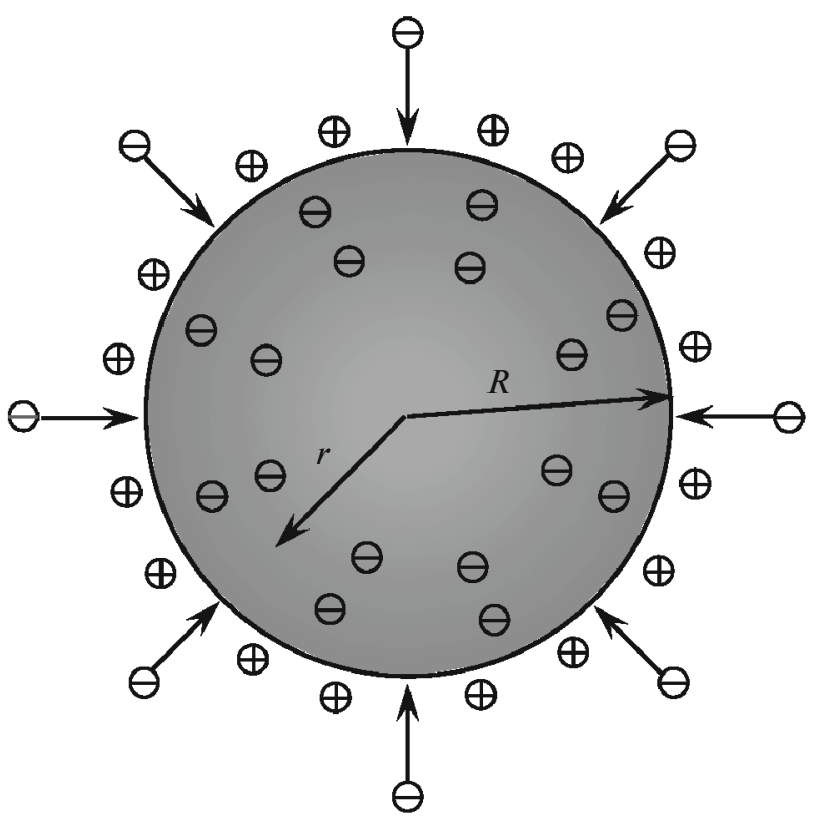

Fig. 1. A model of a satellite in the form of a dielectric ball subjected to isotropic irradiation by relativistic electron fluxes.

mirror coating was applied to the outer surface of one of the hemispheres.

Surkov and Mozgov (2019) studied the effect of the electrification of BLITS type passive dielectric satellites under the influence of the ERB electron flow and calculated the distribution of the electric field inside the satellite. The goal of this study is to generalize the results of that work and to develop a more perfect model of the phenomena, that takes into account the formation of a screening plasma layer on the surface of the satellite and the appearance of radiation-induced conductivity in its bulk.

\section{STATEMENT AND GENERAL SOLUTION OF THE PROBLEM}

Let us consider a nanosatellite model in the form of a rotating dielectric ball moving in an infinite rarefied two-component plasma consisting of electrons and positive ions (Fig. 1). We consider the ball radius $R$ to be small in comparison with the track length of the plasma particles. The ball absorbs flows of relativistic electrons of the ERB, acquiring a negative electric charge $q$. Plasma disturbance by the electric field of this charge leads to the formation of a layer of screening plasma charge around the ball. In a reference frame fixed to the satellite, the electric potential of this field $\varphi$ and the electric charge density $\rho_{e}$ satisfy the Laplace equation $\nabla^{2} \varphi=-\rho_{e} / \varepsilon_{0}$, and the distribution functions of electrons and ions are determined by Boltzmann kinetic equations (Alpert et al., 1964).
Taking into account that the period of ball rotation is on the order of $0.2 \mathrm{~s}$, i.e., it is much less than the characteristic relaxation time of charges in a dielectric (Surkov and Mozgov, 2019), let us simplify the problem by assuming that the electron-flux density vector of the ERB averaged over the period of ball rotation is directed radially everywhere to the ball center and is the same in absolute value at all points of the ball surface. Let $j$ denote the absolute value of the average flux density of electrons in the energy range $(w, w+d w)$. The number of electrons with such energies falling on the ball surface during the time $d t$ is equal to $d^{2} N=4 \pi R^{2} j d t d w$. We assume that, on average, the ERB electrons are injected into the ball uniformly, i.e., the space charge of the ball and its electric field are spherically symmetric. If the asymmetry of the cosmic plasma flowing around the satellite is disregarded, then the electric field potential in the plasma surrounding the ball also depends only on the distance $r$ to the ball center. Therefore, the problem as a whole becomes spherically symmetrical.

The average track length $l$ of charged particles in matter depends on their energy $w$. Let the function $l=l(w)$ defines the given dependence. Suppose that during time $d t$, particles with energies in a given range of values $(w, w+d w)$ penetrate the ball and occupy a spherical layer with a radius of $r=R-l(w)$ and a thickness of $d r=d l(w)$. The further change in the space density of the electric charge is governed by the small, but finite in magnitude, electrical conductivity of the dielectric.

Taking into account the symmetry of the problem, we use a spherical coordinate system with the origin located in the ball center. If we disregard the deceleration time of the incident electrons, assuming that they penetrate the substance almost instantly, then the continuity equation, which determines the principle of electric charge conservation inside the ball, has the form

$$
\frac{\partial \rho_{e}}{\partial t}=-\frac{1}{r^{2}} \frac{\partial}{\partial r}\left(r^{2} J_{r}\right)-\frac{e}{4 \pi r^{2}} \frac{\partial^{2} N}{\partial t \partial l},
$$

where $J_{r}$ is the radial projection of the electric-current density; $\rho_{e}$ is the density of the electric charge; and $e$ is the elementary charge. Substituting the expression for $d^{2} N$ into equation (1), using Maxwell's equation $\rho_{e} /\left(\varepsilon \varepsilon_{0}\right)=\nabla \cdot \mathbf{E}$ and Ohm's law $\mathbf{J}=\sigma \mathbf{E}$, where $\mathbf{E}$ is the electric-field strength; $\varepsilon$ and $\sigma$ are the dielectric constant and conductivity of the substance, and $\varepsilon_{0}$ is the electrical constant, we rewrite equation (1) in the form:

$$
\frac{\partial}{\partial r}\left\{r^{2}\left(\varepsilon \varepsilon_{0} \frac{\partial E_{r}}{\partial t}+\sigma E_{r}\right)\right\}=-e R^{2} j \frac{d w}{d l},
$$


where $E_{r}$ is the radial component of the electric-field strength vector.

When charged particles pass through a dielectric, their kinetic energy is transformed mainly into molecular excitation and ionization of the substance, which is accompanied by the formation of electron-hole pairs and other charged structural defects, and, as a consequence, the appearance of radiation conductivity of the dielectric. For example, according to the laboratory tests and calculations of Rodgers et al. (2000), when Teflon and epoxy samples were irradiated with electrons in an energy spectrum corresponding to the conditions in a geostationary orbit, the radiation conductivity of the first sample exceeded its intrinsic conductivity by three times, and the second exceeded it by two orders of magnitude. In what follows, we assume that the conductivity $\sigma$ in equation (2) includes both intrinsic and radiation conductivity, which depends on the distance to the ball surface.

Let us assume that the ball is surrounded by a twocomponent plasma consisting of electrons and positive, singly charged ions of the same type. The electric field of a charged ball leads to violation of the plasma quasi-neutrality at distances on the order of the Debye radius: $r_{D}=\left\{\varepsilon_{0} k_{B} T_{e} T_{i} /\left[n_{e} e^{2}\left(T_{e}+T_{i}\right)\right]\right\}^{1 / 2}$, where $n_{e}$ is the number density of space plasma; $k_{B}$ is the Boltzmann's constant; and $T_{e}$ and $T_{i}$ are the temperatures of electrons and ions, respectively (Alpert et al., 1964). Using the averaged values of the ionospheric parameters for an altitude of $1500 \mathrm{~km}\left(n_{e} \approx 1.6 \times 10^{10} \mathrm{~m}^{-3}\right.$, $T_{e} \approx 3300 \mathrm{~K}, T_{i} \approx 2050 \mathrm{~K}$ (Novikov et al., 2007)), we obtain $r_{D} \approx 2.1 \mathrm{~cm}$. Considering as an example the BLITS-M nanosatellite, which has the shape of a ball with a radius of $R=11 \mathrm{~cm}$, it can be assumed that a narrow charged plasma layer with a characteristic size $\sim r_{D} \ll R \ll \lambda_{e, i, n}$, is formed around the satellite. Here $\lambda_{e, i, n}$ denotes the mean free path of electrons, ions, and neutral particles, respectively. Outside this layer, the potential takes on the value $\varphi_{1} \approx-0.690 k_{B} T_{e, i} / e$, and then its value tends to zero at $r \rightarrow \infty$ according to asymptotical law: $\varphi \approx-0.237 k_{B} T_{e, i} R^{2} /\left(e r^{2}\right)$ (Alpert et al., 1964). With the numerical values of the parameters given above, we obtain $\varphi_{1} \approx-0.2 \mathrm{~B}$. Further analysis will show that the potential $\varphi_{0}$ of the satellite surface satisfies the relation $e\left|\varphi_{0}\right| \gg k_{B} T_{e, i}$, that is $\left|\varphi_{0}\right| \gg\left|\varphi_{1}\right|$. Thus, the charged plasma layer surrounding the satellite almost completely screens its electric field at distances exceeding $r_{D}$.

Taking into account the narrowness of this layer, one can roughly estimate the energy losses of electrons in the Debye layer as follows: $w_{0} \sim e\left|\varphi_{0}\right| \sim e E_{0} r_{D}$, where $E_{0}$ is the electric field on the satellite surface. Let us assume that the kinetic energy of the incident electrons of the ERB varies from $w_{\min }$ to $w_{\max }$. Taking into account the electron energy losses in the Debye layer and assuming first that $w_{\min }>w_{0}$, we conclude that the energies of electrons reaching the surface of the ball vary within the interval $w_{\min }-w_{0}<w<w_{\max }-w_{0}$. The flux density of these electrons on the ball surface is determined by the function $j=j\left(w+w_{0}, t\right)$.

Let $l_{\max }$ be the maximum average path in the dielectric of incident particles with the maximum energy $w_{\max }-w_{0}$. Due to the spherical symmetry of the problem, the electric-field strength is zero in the region $r<R-l_{\max }$ inside the ball. Taking into account this circumstance, let us integrate equation (2) along the radius from $R-l_{\max }$ to $r$. As a result, we get

$$
\frac{\partial E_{r}}{\partial t}+\frac{\sigma(r, t) E_{r}}{\varepsilon \varepsilon_{0}}=-\frac{e R^{2}}{\varepsilon \varepsilon_{0} r^{2}} \int_{R-l_{\max }}^{r} j\left(w+w_{0}, t\right) \frac{d w}{d l} d r .
$$

The solution to equation (3) with zero initial condition has the form

$$
\begin{aligned}
E_{r}(r, t)= & -\frac{e R^{2}}{\varepsilon \varepsilon_{0} r^{2}} \int_{0}^{t}\left\{\int_{R-l_{\max }}^{r} j\left(w+w_{0}, t^{\prime}\right) \frac{d w}{d l} d r\right\} \\
& \times \exp \left(-\int_{t^{\prime}}^{t} \frac{\sigma\left(r, t^{\prime \prime}\right)}{\varepsilon \varepsilon_{0}} d t^{\prime \prime}\right) d t^{\prime} .
\end{aligned}
$$

In this relation, the function $j\left(w+w_{0}, t\right)$ is equal to the flux density of incident electrons, the track length of which in the ball is equal to the distance $r=l(w)$. If the function $l(w)$ is known, then it can be used to find the implicit dependence of $j$ on the radius $r$. Note that the function $j$ may depend on time if there is a change in external conditions associated with the solar activity, magnetic storms, or other reasons.

\section{STATIONARY DISTRIBUTION OF FIELDS AND SPACE CHARGES}

Let us study the obtained solution for the case in which the flux density of incident electrons $j$ does not depend on time. The characteristic relaxation time of electric charges $\tau=\varepsilon \varepsilon_{0} / \sigma$ in equation (4) ranges from several hours to several days, depending on the type of dielectric and the nature of its conductivity (Surkov and Mozgov, 2019). If a $t \gg \tau$ then solution (4) is simplified. It can be easily derived from equation (3) if we disregard the $E_{r}$ derivative with respect to time and assume that $\sigma$ does not depend on $t$. Then substituting $d r=-d l$ into the integral and passing to the variable of integration $w$, we obtain the stationary distribution of the electric field in the ball:

$$
E_{r}=-\frac{e R^{2}}{\sigma(r) r^{2}} \int_{w(r)}^{w_{\max }-w_{0}} j\left(w+w_{0}\right) d w .
$$


Here, $w(r)$ denotes the kinetic energy of electrons with a pass $R-r$, while $\sigma(r)$ determines the radial distribution of electrical conductivity at $t \gg \tau$.

Equation (5) describes a stationary regime in which the distribution of charges and electric field inside the ball remains constant. In this mode, the flux of ERB electrons incident on the surface and the flux of electrons moving from the volume to the surface due to the electrical conductivity of the ball are equal to each other. The charge accumulating on the ball surface does not affect the field inside the ball due to the spherical symmetry of the problem. In addition, this surface charge is quickly emitted into the surrounding space due to the photoelectric effect and secondary electron-ion emission (Surkov and Mozgov, 2019). Therefore, the field outside the ball is mainly determined by the space charge.

The electron flux density of the ERB can vary widely depending on the electron energy, solar activity, etc. On the daytime side of the ionosphere with moderate solar activity, the dependence of $\log j$ on $\log w$ is approximately linear in an electron energy range from 0.1 to $2-3 \mathrm{MeV}$ (Kuznetsov, 2007). Therefore, the relationship between the quantities $j$ and $w$ can be approximated with an approximate power-law dependence of the form $j(w)=b_{1} w^{-b_{2}}$. For heights of 500-600 km, the electron-flux density $j$ in the energy range of interest decreases from $10^{6}$ to $10^{2} \mathrm{~cm}^{-2} \mathrm{~s}^{-1} \mathrm{MeV}^{-1}$. In this case, the parameters of this dependence have the following values: $b_{1}=2 \times 10^{3} \mathrm{~cm}^{-2} \mathrm{~s}^{-1}$ $\mathrm{MeV}^{b_{2}-1}, b_{2}=2.7$ (Surkov and Mozgov, 2019). Substituting this relation into integral (5) and performing integration, we obtain

$$
E_{r}=-\frac{e R^{2} b_{1}}{\sigma(r) r^{2}\left(b_{2}-1\right)}\left\{\frac{1}{\left[w(r)+w_{0}\right]^{b_{2}-1}}-\frac{1}{w_{\max }^{b_{2}-1}}\right\} .
$$

The electric field caused by the flux of protons or other charged particles falling on the satellite surface can be described in a similar way. However, since the electron-flux density is approximately three orders of magnitude higher than the proton flux density in low orbits (Kuznetsov, 2007), we will further disregard the contribution of the proton flux.

The track length in matter of electrons with energies on the order of $0.1-10 \mathrm{MeV}$ is determined with the following empirical formula (Weber, 1964):

$$
l(w)=\frac{a_{1} w}{\rho_{m}}\left(1-\frac{a_{2}}{1+a_{3} w}\right),
$$

where $a_{1}=0.55 \mathrm{~g} \mathrm{~cm}^{-2} \mathrm{MeV}^{-1}, a_{2}=0.9841$ and $a_{3}=$ $3 \mathrm{MeV}^{-1}$ are empirical parameters; $\rho_{m}$ is the density of the substance, measured in $\mathrm{g} / \mathrm{cm}^{3}$; and the electron energy $w$ is measured in MeV. Replacing $l(w)$ with $r$ in equation (7) and expressing from it $w$, we get the following dependence:

$$
\begin{gathered}
w(r)=\frac{1}{2}\left[\frac{\rho_{m}(R-r)}{a_{1}}-\frac{1-a_{2}}{a_{3}}\right] \\
+\left\{\frac{1}{4}\left[\frac{\rho_{m}(R-r)}{a_{1}}-\frac{1-a_{2}}{a_{3}}\right]^{2}+\frac{\rho_{m}(R-r)}{a_{1} a_{3}}\right\}^{1 / 2} .
\end{gathered}
$$

If $w_{\min }>w_{0}$, then equations (7) and (8) are applicable in the range $R-l_{\max }<r<R-l_{\text {min }}$.

The radiation conductivity arising due to the action of a flux of ERB electrons falling on the ball, reaches a maximum value, $\sigma_{r}$, on its surface. In the direction of the ball interior, it decreases on a characteristic scale $\bar{l}$, which is equal to the average track length of electrons in the ball. For radii $r$ satisfying the condition $R-r \gg \bar{l}$, the radiation conductivity becomes less than its intrinsic conductivity $\sigma_{0}$. Since $R \gg \bar{l}$ and $\sigma_{r} \gg \sigma_{0}$, we use the following approximation:

$$
\sigma(r)=\sigma_{0}+\left(\sigma_{r}-\sigma_{0}\right) \exp \{-(R-r) / l\},
$$

where $\bar{l}$ is the mean range of electrons over the flux:

$$
\bar{l}=\int_{w_{\min }}^{w_{\max }} l\left(w-w_{0}\right) j(w, t) d w\left(\int_{w_{\min }}^{w_{\max }} j(w, t) d w\right)^{-1} .
$$

Equation (10) is applicable if $w_{\min }>w_{0}$. If the opposite inequality holds, then a portion of the incident electrons will not be able to reach the ball surface due to electrostatic repulsion in the Debye layer surrounding the ball. The ball surface will only be reached by those electrons with an energy exceeding $w_{0}$. Therefore, in this case, it is necessary to replace $w_{\min }$ with $w_{0}$ in equation (10).

We find the average track length by substituting the relations for $j(w)$ and $l(w)$ into equation (10). After some rearranging, we obtain

$$
\begin{gathered}
\bar{l}=\frac{a_{1}\left(b_{2}-1\right)}{\rho_{m}\left(w_{\min }^{1-b_{2}}-w_{\max }^{1-b_{2}}\right)} \times \\
\times \int_{w_{\min }}^{w_{\max }}\left(w-w_{0}\right)\left\{1-\frac{a_{2}}{1+a_{3}\left(w-w_{0}\right)}\right\} \frac{d w}{w^{b_{2}}} .
\end{gathered}
$$

Substituting functions $w(r)$ and $\sigma(r)$ into equation (6), we obtain the distribution of the electric field in the ball over the radius. If $w_{\min }>w_{0}$, then this distribution is applicable in the range $R-l_{\max }<r<R-l_{\min }$, where $l_{\max }$ and $l_{\min }$ are the track lengths of electrons with energies of $w_{\max }-w_{0}$ and $w_{\min }-w_{0}$, respectively. If $w_{\min }<w_{0}$, then formally $l_{\text {min }}=0$. There are no electrons in the narrow layer bounded by radii $R-l_{\min }<r<R$, since the model does not consider 


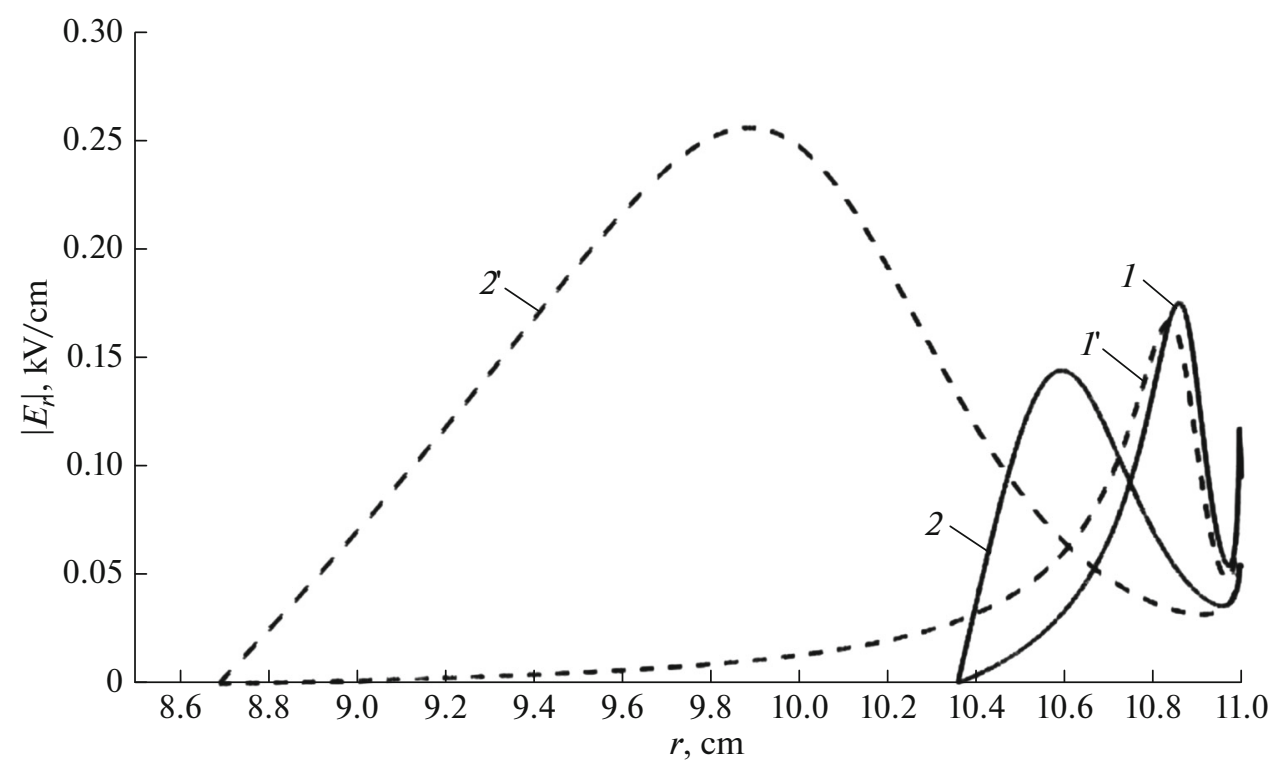

Fig. 2. Dependence of the absolute value of the radial electric field on the distance to the ball center calculated for the dielectric radiation conductivity $\sigma_{r}=10^{-14} \mathrm{~S} / \mathrm{m}$. Graphs shown with lines 1 and $l^{\prime}$ correspond to low orbits while graphs 2 and 2 correspond to higher orbits. The maximum electron energy is $3 \mathrm{MeV}$ (lines 1 and 2) or $10 \mathrm{MeV}$ (lines $l^{\prime}$ and 2'). The graphs shown with lines 2 and $2^{\prime}$ are reduced by 50 times.

electrons with initial energies lower than $w_{\min }$. The absolute value of the electric field on the satellite surface is then $E_{0} \approx \varepsilon\left|E_{r}\right|$, where $E_{r}$ in equation (6) is taken at the point $r=R-l_{\min }$. The energy $w_{0} \sim e r_{D} E_{0}$ lost by incident electrons in the Debye layer is estimated as follows:

$$
w_{0} \approx \frac{e^{2} b_{1} r_{D} \varepsilon}{\sigma_{r}\left(b_{2}-1\right)}\left(\frac{1}{w_{\min }^{b_{2}-1}}-\frac{1}{w_{\max }^{b_{2}-1}}\right) .
$$

It should be noted that, if $w_{\min }<w_{0}$, then $w_{\text {min }}$ needs to be replaced with $w_{0}$ in equations $(10)-(12)$. In this case, equation (12) determines the implicit dependence $w_{0}$ on the problem parameters.

Let us carry out numerical estimates of the obtained values. Laboratory tests and calculations show that when dielectric samples are irradiated by electrons with energies corresponding to the conditions in a geostationary orbit, their conductivity can increase from several times to two to three orders of magnitude (Tyutnev et al., 2015). As an example, we use the parameters of quartz glass : $\varepsilon=3.7, \sigma_{0}=10^{-16} \mathrm{~S} / \mathrm{m}$, and $\rho_{m}=2.3 \mathrm{~g} / \mathrm{cm}^{3}$ (Babichev et al., 1991). Using the parameters given above as well as $\sigma_{r}=10^{-14} \mathrm{~S} / \mathrm{m}$, $w_{\text {min }}=0.1 \mathrm{MeV}$, and $w_{\text {max }}=3-10 \mathrm{MeV}$, we obtain the following estimates: $w_{0}=0.73 \mathrm{keV}, E_{0}=0.56 \mathrm{kV} / \mathrm{cm}$, $l_{\text {min }}=0.06 \mathrm{~mm}$, and $l_{\max }=0.65-2.3 \mathrm{~cm}$. In this case, the energy loss of incident electrons in the plasma layer can be disregarded in comparison with the initial electron energy.

The dependence $E_{r}(r)$, as calculated with the parameters given above, is illustrated in Fig. 2 with lines 1 and $l^{\prime}$ for cases in which $w_{\max }=3 \mathrm{MeV}$ and $w_{\max }=10 \mathrm{MeV}$, respectively. Since $E_{r}<0$, the graphs of the function $\left|E_{r}\right|$ are shown for convenience. As is seen from these graphs, one of the maxima is located near the surface at a distance $l_{\min }$ from it. The second maximum, which has larger value, is located deeper. For line graph 1 , the maximum field value of $0.175 \mathrm{kV} / \mathrm{cm}$ is achieved at $r=10.86 \mathrm{~cm}$. For the graph shown with line 1 , these values are $0.166 \mathrm{kV} / \mathrm{cm}$ and $10.83 \mathrm{~cm}$, respectively. These values are very close, i.e., for these parameters, the magnitude and position of the electric-field maximum have almost no dependence on the magnitude $w_{\max }$.

For orbits at heights $\sim 1500 \mathrm{~km}$, the flux density of ERB electrons with the energies of interest varies within the range of the order of $10^{4}-10^{7} \mathrm{~cm}^{-2} \mathrm{~s}^{-1} \mathrm{MeV}^{-1}$. For this energy range, the parameters determining the dependence $j(w)$ have the following values: $b_{1}=3.2 \times 10^{5} \mathrm{~cm}^{-2} \mathrm{~s}^{-1} \mathrm{MeV}^{b_{2}-1}$, and $b_{2}=1.5$ (Surkov and Mozgov, 2019). In this case, we find that $w_{0}=21-23 \mathrm{keV}$ and $E_{0}=11 \mathrm{kV} / \mathrm{cm}$, i.e., the amendment $w_{0}$ becomes essential. For this case the dependence $\left|E_{r}\right|$ on $r$ is shown in Fig. 2 by lines 2 and 2', which correspond to the values $w_{\max }=3$ and $10 \mathrm{MeV}$, 


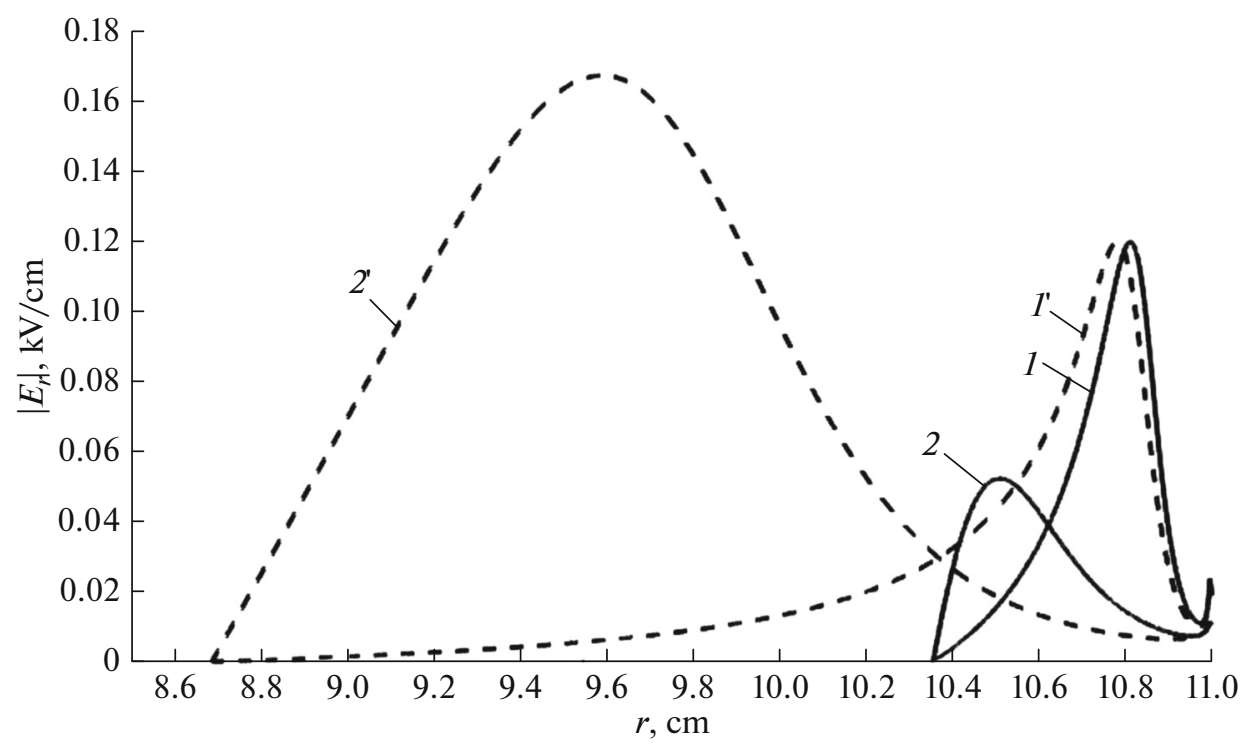

Fig. 3. The same as in Fig. 2, but for $\sigma_{r}=5 \times 10^{-14} \mathrm{~S} / \mathrm{m}$.

respectively. For convenience, the values of $\left|E_{r}\right|$ are reduced by 50 times. These graphs are also nonmonotonous. For graph shown with line 2 the maximum value of $7.19 \mathrm{kV} / \mathrm{cm}$ is achieved at $r=10.60 \mathrm{~cm}$. For graph 2' these values are equal to $12.8 \mathrm{kV} / \mathrm{cm}$ and $r=9.89 \mathrm{~cm}$, respectively. Thus, at the aforementioned electron-flux density, the magnitude and position of the maximum of the graphs, as well as the field penetration depth $(0.65 \mathrm{~cm}$ and $2.3 \mathrm{~cm}$ for the lines 2 and 2', respectively), depend significantly on the value of $w_{\max }$.

Now let us analyze how the parameter $\sigma_{r}$, which determines the maximum of the radiation conductivity of the dielectric, affects the electric field. Figure 3 shows the absolute value of the radial component of the electric field versus the radius for the same parameters that were used for Fig. 2, but for the value $\sigma_{r}=5 \times 10^{-14} \mathrm{~S} / \mathrm{m}$. First of all, we note that the graphs are still non-monotonic in character. A comparison with Fig. 2 shows that an increase in radiation conductivity $\sigma_{r}$ leads to a decrease in the maxima of the electric field and a shift of their positions towards the ball center. For example, for lines 1 and 2, the maxima decrease to values of 0.12 and $2.61 \mathrm{kV} / \mathrm{cm}$, respectively. The coordinates of the corresponding maxima, $r=10.81$ and $10.51 \mathrm{~cm}$, are located slightly closer to the ball center as compared to Fig. 2.

\section{DISCUSSION}

Note that the results of the study only provide estimates of the effects, since the simplest model of a spherical satellite is used, which does not take into account the dependence of material properties on temperature, the daily and seasonal variations in the flow of ERB electrons incident on the satellite, and a number of other factors. Nevertheless, the analytical results obtained in this study make it possible to analyze the process of the satellite electrification depending on various parameters, including the radiation conductivity of dielectrics.

The calculation results show that the electric field and charges are distributed near the satellite surface in a layer with a thickness of 0.65 to $2.3 \mathrm{~cm}$ depending on the maximum energy of the ERB electrons. The nonmonotonous dependences of $E_{r}(r)$, shown in Figs 2 and 3, differ from the previous study (Surkov and Mozgov, 2019), in which the absolute value of $E_{r}$ increased monotonically towards the ball surface. One important factor affecting the non-monotonic behavior of the function $E_{r}(r)$ is the dependence of the radiation conductivity of the dielectric on the radius, which was not taken into account earlier (Surkov and Mozgov, 2019).

To gain a better understanding of the results obtained above, we will make a number of simplifications in equations (6)-(8). Assuming that $a_{2} /\left(1+a_{3} w\right) \ll 1$, we omit the corresponding term in equation (7). It then follows from equation (8) that $w \approx x \rho_{m} / a_{1}$, where $x=R-r$ is the distance to the ball surface. Analysis of the numerical values of the parameters shows that, near the maximum point $\left|E_{r}\right|$, first, we can disregard the second term in square brackets in equation (6), and, second, we can assume that the $R^{2} / r^{2} \approx 1$. Equation (6) is then simplified to the form 


$$
E_{r}(x) \approx \frac{e b_{1}\left(x \rho_{m} / a_{1}+w_{0}\right)^{1-b_{2}}}{\left(1-b_{2}\right)\left[\sigma_{0}+\left(\sigma_{r}-\sigma_{0}\right) \exp (-x / \bar{l})\right]} .
$$

The function in the numerator of equation (13) determines the dependence of the electric field on the track length of the ERB electrons in the satellite body. This function decreases with distance $x$, since $b_{2}>1$. If the conductivity $\sigma$ is a constant value, then the electric field will also decrease with distance in accordance with the earlier results (Surkov and Mozgov, 2019). However, since the electrical conductivity in the denominator of equation (13) also decreases, then the function $E_{r}(x)$ may be non-monotonic. To find the extreme points, we take the derivative of equation (13) with respect to $x$. Equating it to zero, we obtain an implicit equation that determines the extreme points:

$$
\exp \left(\frac{x}{\bar{l}}\right)=\left(\frac{\sigma_{r}}{\sigma_{0}}-1\right)\left[\frac{\left(x+a_{1} w_{0} / \rho_{m}\right)}{\left(b_{2}-1\right) \bar{l}}-1\right] .
$$

In this relation $\bar{l}$ depends on $w_{\max }, b_{2}$, and other problem parameters in accordance with equation (11). At given above parameter, equation (14) has two roots, one of which determines the minimum and the other determines the maximum of the function $E_{r}(x)$. For example, for parameters corresponding to lines $l^{\prime}$ and $2^{\prime}$ in Fig. 2, the solution of equation (14) gives the following approximate coordinates of the maximum points: $r_{\max 1}=R-x_{\max 1} \approx 10.85 \mathrm{~cm}$ for the first graph and $r_{\max 2} \approx 9.61 \mathrm{~cm}$ for the second graph. These maximum points are close to the coordinates of the corresponding maxima of the graph shown with lines 1' and 2'.

Analysis of the distribution of space charges density $\rho_{e}$ shows that $\rho_{e}<0$ in the interior $R-l_{\max }<r<r_{0}$, where $r_{0}$ approximately coincides with the maximum coordinate of the function $E_{r}$. However, in the area $r>r_{0}$, where the derivative of $E_{r}$ changes sign, a positively charged layer appears. Physically, this is due to the fact that the function $\rho_{e}$ depends not only on the distribution of electrons embedded in the dielectric but also on $\nabla \sigma$.

Thus, the features of the spatial distributions of the electric field and charges are caused by the fact that the number density of embedded electrons and the radiation conductivity of the dielectric increase with radius. The "competition" of these two tendencies, one of which increases the field while the other decreases it, brings into existence the maximum of the $E_{r}$ function near the satellite's surface. The coordinates of the position of this maximum and its value substantially depend on both the parameters of the ERB electron flux and the radiation conductivity of the satellite material.

Calculations show that an increase in the flux density and energy of the ERB electrons leads to an increase in the electric field in the dielectric, while the magnitude of the field maximum is approximately two orders of magnitude lower than the breakdown threshold of the dielectric under laboratory conditions. However, experiments on board the CRESS spacecraft (Frederickson et al., 1992; Akishin et al., 2007) showed that a breakdown in a space-charged dielectric under space conditions occurs when the electrons fluence is 2-3 orders of magnitude less than the threshold value at which the dielectric breakdown is observed under laboratory conditions. Therefore, one may assume that electrical microbreakdowns can occur in the surface layer of a dielectric, especially near inhomogeneities, microcracks, and surface irregularities, where the local electric field is greater than the average value. Breakdown can be initiated, for example, by galactic or solar cosmic protons with high energies, the track length of which is comparable to or exceeds the satellite diameter.

The local heating of a substance during microbreakdowns can be accompanied by thermal deformations and microdestructions of the substance, which will accumulate over time. From this point of view, prolonged irradiation of a dielectric satellite with EPB electrons is similar to the action of prolonged mechanical loads, which lead to the fatigue failure of materials.

\section{CONCLUSIONS}

The results of model calculations show that irradiation of a dielectric satellite with EPR electrons with energies $0.1-10 \mathrm{MeV}$ leads to the appearance of an electric field and charges in the surface layer of a dielectric with a thickness $\sim 0.65-2.3 \mathrm{~cm}$. The incident electrons lose part of their energy in the Debye shielding layer formed on the outer surface of the satellite due to the polarization of the cosmic plasma in the electric field of the satellite. The energy loss of electrons, according to estimates, is $1-23 \mathrm{keV}$ depending on the altitude of the satellite's orbit. As a result of this effect, the mean electron track length in the dielectric decreases.

Another important effect is an increase in the electrical conductivity of the satellite surface layers due to the material ionization produced by the flow of incident electrons. Calculations that take into account the effect of the radiation conductivity of the dielectric show that the dependence of the electric field on the radius is nonmonotonic. From the analysis of the obtained solution, it follows that this property of the $E_{r}(r)$ dependence is due to the inhomogeneity of the radiation conductivity of the dielectric.

With an increase in the flux density and energy of the ERB electrons, the maximum of the electric field increases, and its position shifts from the surface into the interior of the satellite. For the selected parameter values and an orbit height of $\sim 1500 \mathrm{~km}$, the maximum electric field is estimated as $2.5-7 \mathrm{kV} / \mathrm{cm}$ at a maxi- 
mum electron energy of $3 \mathrm{MeV}$ and $8-13 \mathrm{kV} / \mathrm{cm}$, if $w_{\max }=10 \mathrm{MeV}$. Although these values are approximately two orders of magnitude less than the dielectric breakdown threshold, local electric discharges can be expected near inhomogeneous inclusions and irregularities on the dielectric surface. The probability of dielectric microbreakdown increases during periods of maximum solar activity.

Despite the evaluative nature of the study, the results of this work can be applied to low-orbit spherical nanosatellites, such as BLITS and BLITS-M.

\section{FUNDING}

The study was partially supported by the Russian Foundation for Basic Research, project no. 18-05-00108.

\section{OPEN ACCESS}

This article is licensed under a Creative Commons Attribution 4.0 International License, which permits use, sharing, adaptation, distribution and reproduction in any medium or format, as long as you give appropriate credit to the original author(s) and the source, provide a link to the Creative Commons licence, and indicate if changes were made. The images or other third party material in this article are included in the article's Creative Commons licence, unless indicated otherwise in a credit line to the material. If material is not included in the article's Creative Commons licence and your intended use is not permitted by statutory regulation or exceeds the permitted use, you will need to obtain permission directly from the copyright holder. To view a copy of this licence, visit http://creativecommons.org/licenses/by/4.0/.

\section{REFERENCES}

Akishin, A.I. and Novikov, L.S., Elektrizatsiya kosmicheskikh apparatov (Electrification of Space Vehicles), Moscow: Znanie, 1985.

Akishin, A.I., Novikov, L.S., Makletsov, A.A., and Mileev, V.N., Ob"emnaya elektrizatsiya dielektricheskikh materialov kosmicheskikh apparatov, in Model' Kosmosa (Model of the Cosmos), vol. 2: Vozdeistvie kosmicheskoi sredy na materialy i oborudovanie kosmi-cheski$k h$ apparatov (Space Impact on the Matter and Equipment of Space Vehicles), Panasyuk, M.I. and Novikov, L.S., Eds., Moscow: KDU, 2007, pp. 315-344.

Al'pert, Ya.L., Gurevich, A.V., and Pitaevskii, L.P., Iskusstvennye sputniki v razrezhennoi plazme (Artificial Satellites in Rarified Plasma), Moscow: Nauka, 1964.

Babichev, A.P., Babushkina, N.A., Bratkovskii, A.M., et al., Fizicheskie velichiny (Physical Quantities), Grigor'ev, I.S. and Meilikhov, E.Z., Eds., Moscow: Energoatomizdat, 1991.

Bezrodnykh, I.P., Tyutnev, A.P., and Semenov, V.T., Radiatsionnye effekty $v$ kosmose (Radiation Effects in Space), vol. 2: Vozdeistvie kosmicheskoi radiatsii na elektrotekhnicheskie materialy (Cosmic Radiation Effect on Electrical Supplies), Moscow: VNIIEM, 2016.
De Forest, S.E., Spacecraft charging at synchronous orbit, J. Geophys. Res., 1972, vol. 77, no. 4, pp. 651-659.

Frederickson, A.R., Holeman, E.G., and Mullen, E.G., Characteristics of spontaneous electrical discharges of various insulators in space radiations, IEEE Trans. Sci., 1992, vol. 39, no. 6, pp. 1773-1982.

Kucharski, D., Kirchner, G., Lim, H.-C., et al., Optical response of nanosatellite BLITS measured by the Graz 2 kHz SLR system, Adv. Space Res., 2011, vol. 48, no. 8, pp. $1335-1340$. https://doi.org/10.1016/j.asr.2011.06.016

Kuznetsov, N.V., Radiation conditions in space vehicle orbits, in Model' Kosmosa (Model of the Cosmos), vol. 1: Fizicheskie usloviya $v$ kosmicheskom prostranstve (Physical Conditions in Space), Panasyuk, M.I. and Novikov, L.S., Eds., Moscow: KDU, 2007, pp. 627-641.

Lai, S.T., Fundamentals of Spacecraft Charging: Spacecraft Interactions with Space Plasmas, Princeton, N.J.: Princeton University Press, 2011.

Lai, S.T. and Cahoy, K., Spacecraft charging, in Encyclopedia of Plasma Technology, Taylor and Francis, 2017, pp. 1352-1366. https://doi.org/10.1081/E-EPLT-120053644

Lai, S.T., Cahoy, K., Lohmeyer, W., Carlton, A., Aniceto, R., and Minow, J., Deep dielectric charging and spacecraft anomalies, Extreme Events Geospace, 2018, pp. 419432. https://doi.org/10.1016/B978-0-12-812700-1.00016-9.

Novikov, L.S., Mileev, V.N., Krupnikov, K.K., and Makletsov, A.A., Electrification of space vehicles in the magnetospheric plasma, in in Model' Kosmosa (Model of the Cosmos), vol. 2: Vozdeistvie kosmicheskoi sredy na materialy $i$ oborudovanie kosmicheskikh apparatov (Space Impact on the Matter and Equipment of Space Vehicles), Panasyuk, M.I. and Novikov, L.S., Eds., Moscow: KDU, 2007, pp. 236-275.

Novikov, L.S., Makletsov, A.A., and Sinolits, V.V., Modeling of spacecraft charging dynamics using COULOMB-2 code, IEEE Trans. Plasma Sci., 2017, vol. 45, no. 8, pp. 1915-1918. https://doi.org/10.1109/TPS.2017.2720595

Rodgers, D.J., Ryden, K.A., Wrenn, G.L., Latham, P.M., Sorensen, J., and Levy, L., An engineering tool for the prediction of internal dielectric charging, in Proc. 6th Spacecraft Charging Technology Conference, 2000, pp. 125-130, AFRL-VS-TR-20001578.

Surkov, V.V. and Mozgov, K.S., Effects of the action of particle fluxes and geomagnetic variations on low-orbital spherical satellites, Cosmic Res., 2019, vol. 57, no. 4, pp. 252-260.

https://doi.org/10.1134/S0010952519040075

Tyutnev, A., Saenko, V., Pozhidaev, E., and Ikhsanov, R., Experimental and theoretical studies of radiation-induced conductivity in spacecraft polymers, IEEE Trans. Plasma Sci., 2015, vol. 43, no. 9, pp. 2915-2924. https://doi.org/10.1109/TPS.2015.2403955

Vasil'ev, V.P., Path to accuracy, Ross. Kosmos, 2018, vol. 2, no. 145 , pp. $10-14$.

Weber, K.-H., Eine einfache Reichweite-Energie-Beziehung für Elektronen im Energiebereich von $3 \mathrm{keV}$ bis $3 \mathrm{MeV}$, Nucl. Instrum. Methods, 1964, vol. 25, pp. 261-264. 